\title{
Note sur la présence de Hardya helgae Nickel, Holzinger \& Remane 2017 en Belgique (Hemiptera, Cicadellidae)
}

\author{
Jean-Yves Baugnée
}

Service public de Wallonie, Département de l'Étude du Milieu Naturel et Agricole, Direction de la Nature et de l'Eau, Avenue de la Faculté, 22, B-5030 Gembloux.

E-mail: jybaugnee@gmail.com

Reçu le 5 février 2021, accepté le 15 mars 2021.

La cicadelle Hardya helgae Nickel, Holzinger \& Remane 2017 est signalée pour la première fois de Belgique où elle a été récoltée entre Rochefort et Eprave (province de Namur) les 14.vii.2014, 21.iii.2019 et 14.vi.2019. Cette espèce décrite récemment du sud-ouest de l'Allemagne, puis signalée de l'est de la France et du Grand-Duché de Luxembourg, était auparavant confondue avec Hardya tenuis (Germar 1821). H. helgae est confiné aux pelouses calcaires et se développe sur la graminée Bromus erectus Huds. L'occurrence de $H$. tenuis en Belgique est remise en question du fait qu'elle repose seulement sur deux spécimens du $19^{\text {ème }}$ siècle dont la révision montre qu'ils correspondent plutôt à $H$. helgae.

Mots-clés: Hardya, cicadelle, Auchenorhynques, pelouse calcicole, Bromus erectus, faunistique, Région Wallonne

The leafhopper Hardya helgae Nickel, Holzinger \& Remane 2017 is recorded for the first time from Belgium where it was found at Rochefort and Eprave (province of Namur) on 14.vii.2014, 21.iii.2019 and 14.vi.2019, respectively. This species recently described from southwestern Germany, then recorded from eastern France and the Great Duchy of Luxembourg, was previously confused with Hardya tenuis (Germar 1821). It is confined to calcareous grasslands and grows on the grass Bromus erectus Huds. The occurrence of $H$. tenuis in Belgium is questioned because it is based only on two specimens from the 19th century, the revision of which shows that they correspond more to H. helgae.

Keywords: Hardya, leafhopper, Auchenorrhyncha, calcareous grassland, Bromus erectus, faunistics, Walloon Region

\section{INTRODUCTION}

Les Cicadellidae, généralement dénommés « cicadelles » en langue française (ou encore « leafhoppers » en anglais, «dwergcicaden » en néerlandais, ...) constituent la famille la plus riche de l'ensemble des Hémiptères avec plus de 25000 espèces décrites à travers le monde (Garrouste, 2015) dont plus de 300 sont répertoriées au Benelux (e.a. Den Bieman et al., 2011; Lock, 2019). Classés le plus souvent dans le clade des Auchénorhynques (Fulgoromorpha + Cicadomorpha), assemblage pratique mais probablement artificiel et dont la position phylogénétique demeure encore très discutée (voir e.a. Bourgoin \& Campbell, 2002 ; Forero, 2008), ces insectes se caractérisent par leurs pattes postérieures allongées et modifiées pour le saut et dont le tibia, plus ou moins aplati et caréné, est pourvu d'une à plusieurs rangées d'épines mobiles. Les ailes antérieures des adultes, généralement bien développées, lisses et glabres, plus ou moins coriacées, translucides ou non et de coloration très variée, montrent néanmoins un aspect relativement homogène, d'où l'ancien terme homoptère longtemps utilisé pour regrouper les pucerons, cicadelles et cochenilles. Pour la plupart, les cicadelles sont très agiles et volent fort aisément, ce qui complique souvent leur observation et leur capture. 
Toutes les espèces, aussi bien au stade adulte que durant leur développement larvaire, s'alimentent de sève végétale qu'elles ponctionnent grâce à leurs organes buccaux transformés en rostre piqueur-suceur. Ces insectes sont ainsi étroitement liés aux végétaux, d'autant que beaucoup de représentants se révèlent, du point de vue trophique, fortement spécialisés quant aux plantes hôtes exploitées (Nickel, 2003).

En Belgique, les cicadelles ont toujours joui d'une faible popularité auprès des entomologistes et ont été l'objet d'un nombre restreint de publications, ce qui explique amplement l'état lacunaire de nos connaissances. Le catalogue bibliographique de Van Stalle (1989) constitue le principal travail de référence, partiellement mis à jour par Den Bieman et al. (2011) dans le cadre de l'adaptation pour le Benelux de l'ouvrage de Biedermann \& Niedringhaus (2004).

La publication, au cours des trois dernières décennies, d'excellents guides illustrés (e.a. Remane \& Wachmann, 1993 ; Kunz et al., 2011 ; Mühlethaler et al., 2019), a certainement encouragé davantage de personnes à s'intéresser à ces insectes passionnants, de même d'ailleurs que l'avènement des forums naturalistes sur internet et de la science participative qui contribuent à améliorer notablement les connaissances par l'apport de données de natures très diverses.

La présente note s'inscrit dans le cadre de l'actualisation du catalogue des Auchénorhynques de Belgique. Elle concerne le genre Hardya Edwards, 1922 et plus précisément Hardya helgae Nickel, Holzinger \& Remane 2017 (figure 1), une nouvelle espèce décrite d'Allemagne que nous avons détecté également en Belgique. Comme l'ont bien rappelé Nickel et al. (2017), les Hardya sont de petites cicadelles brunâtres, actuellement réunies au sein de la tribu des Athysanini de la sous-famille des Deltocephalinae (Zahniser \& Dietrich, 2013), dont onze espèces ont été décrites à travers le monde et cinq sur le continent européen (Jach, 2013), outre H. helgae non encore enregistré sur Fauna Europaea.

Les principaux caractères diagnostics se trouvent essentiellement dans la conformation des genitalia mâles et en particulier les lames génitales de forme largement triangulaire et à bords internes divergents, les lobes du pygophore pourvus d'un peigne épineux, l'édéage à base large et à tige étroite et plus ou moins recourbée vers l'extrémité, les styles fortement tronqués-dilatés dans leur moitié basale et effilés à leur extrémité apicale. $\mathrm{P}$

Passant généralement pour des cicadelles assez rares, les Hardya affectionnent les lieux ouverts et plus ou moins arides où elles évoluent sur diverses graminées (Poaceae). Hardya tenuis (Germar 1821) est le seul représentant signalé auparavant de Belgique (Fagel, 1949; Van Stalle, 1989), mais, comme nous le verrons plus loin, son occurrence réelle est remise en question. 


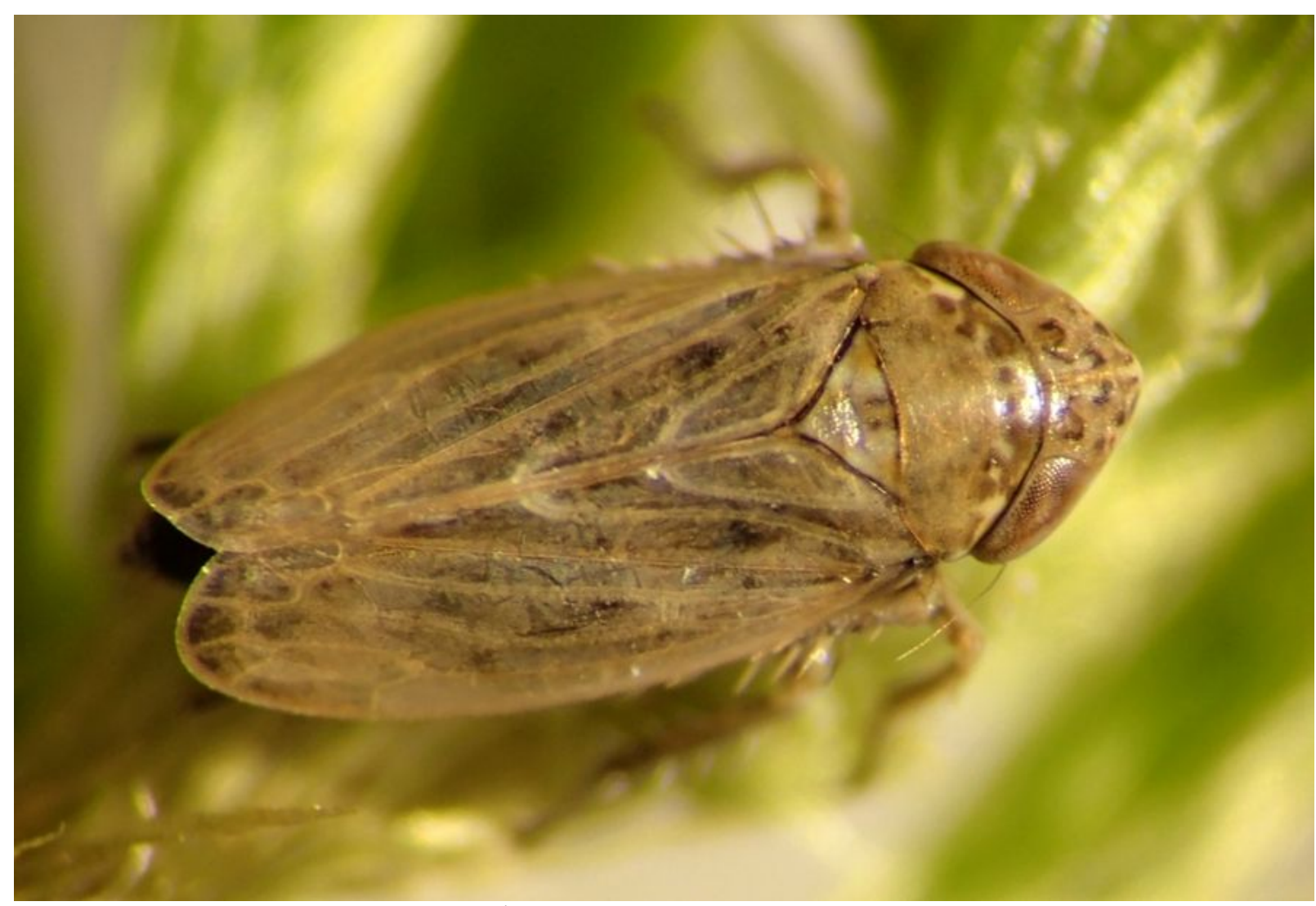

Figure 1: Hardya helgae, ô, 2,8 mm. Eprave, 21.iii.2019 (photo J.-Y. Baugnée).

\section{HARDYA HELGAE EN BELGIQUE}

Dans leur article original, Nickel et al. (2017) indiquent que leur nouvelle espèce Hardya helgae est très proche de $H$. tenuis si ce n'est la longueur du corps des adultes nettement inférieure (mâles 2,8-3,1 mm et femelles 3,0-3,4 mm, contre respectivement 3,5-3,8 $\mathrm{mm}$ et $3,6-4,0 \mathrm{~mm}$ pour $H$. tenuis) et quelques différences dans les pièces génitales du mâle. En outre, si les plantes hôtes des Hardya sont toujours des graminées, elles sont bien distinctes d'une espèce à l'autre: Bromus erectus Huds. pour $H$. helgae et Festuca gr. ovina L. pour H. tenuis.

L'occurrence de $H$. helgae dans plusieurs régions limitrophes et la relative fréquence de sa plante nourricière concouraient à la probabilité de sa découverte sur le territoire belge et nécessitait la révision du matériel disponible. De fait, le réexamen de spécimens récoltés en 2014 sur une pelouse sèche de la région de Rochefort, et initialement déterminés comme $H$. tenuis, nous conduit à reconnaître qu'ils appartiennent en réalité à $H$. helgae. De nouvelles prospections menées en 2019 dans ce même secteur ont permis la collecte de divers autres spécimens de cette espèce.

Matériel examiné : Belgique, province de Namur : Rochefort, Tige d'Eprave, réserve naturelle Natagora (UTM: FR5758; altitude $185 \mathrm{~m}$ ), 20 §̊ㅇ, 14.vii.2014. Eprave, Tige d'Eprave, pelouse ouest (UTM: FR5557; altitude $175 \mathrm{~m}), 2 \hat{\jmath}, 3$ ㅇ, 21.iii.2019. Eprave, Tige d'Eprave - Les Gives, réserve naturelle domaniale (UTM: FR5658 ; altitude $180 \mathrm{~m}$ ), 11수, 3 +, 3 larves, 14.vi.20191.

Toutes les cicadelles ont été récoltées au filet fauchoir dans trois stations dispersées sur près de deux kilomètres d'un bout à l'autre du site du Tige d'Eprave qui s'étire au sud de la route du Tige (ou rue

\footnotetext{
${ }^{1}$ Toutes ces données ont été saisies sur le portail d'encodage du SPW-DEMNA: http://observatoire.biodiversite.wallonie.be/encodage/
} 
Pêcheron) reliant Eprave à Rochefort. Il s'agit d'une zone bocagère à haute valeur paysagère et biologique constituée d'une mosaïque de pelouses calcicoles, de pâtures sèches, de prairies maigres de fauche, de fourrés et de haies, dans le prolongement sud de la plaine de Behotte. Faisant partie du site Natura 2000 BE35025 dénommé "La Famenne entre Eprave et Havrenne", le Tige d'Eprave est également repris à l'inventaire des sites de grand intérêt biologique de la Région wallonne ${ }^{2}$, tandis que plusieurs parcelles bénéficient du statut de réserve naturelle.

La première station, prospectée en juillet 2014, est localisée au sein d'une parcelle gérée par l'association Natagora, à l'extrémité orientale du site, en contrebas de la rue du Tige. C'est une prairie thermophile parsemée de nombreux buissons et entretenue par pâturage bovin. La strate herbacée, très riche, regroupe aussi bien des espèces des pelouses calcicoles que des espèces des prairies maigres et de fauche, notamment Bromus erectus Huds., Festuca lemanii Bastard, Helianthemum nummularium (L.) Mill., Avenula pubescens (Huds.) Dumort., Briza media L., Galium verum L., Brachypodium pinnatum P. Beauv., Teucrium chamaedrys L., Genista tinctoria L., Ononis spinosa L., Knautia arvensis (L.) Coult., Centaurea scabiosa L., Himantoglossum hircinum (L.) Spreng., Scabiosa columbaria L., etc.

La seconde station (figure 2), située sur un terrain privé dans la portion ouest du site, correspond à son point le plus aride : il s'agit d'un versant écorché exposé au sud-est, couvert par une pelouse très sèche largement dominée par Bromus erectus Huds., accompagné d'autres plantes caractéristiques comme Sanguisorba minor Scop., Sedum album L., Thymus pulegioides L., Arabis hirsuta (L.) Scop., Thlaspi perfoliatum L., Globularia bisnagarica L., Echium vulgare L., Festuca lemanii Bastard, Potentilla neumanniana Rchb., Carex flacca Schreb., Hippocrepis comosa L., etc.

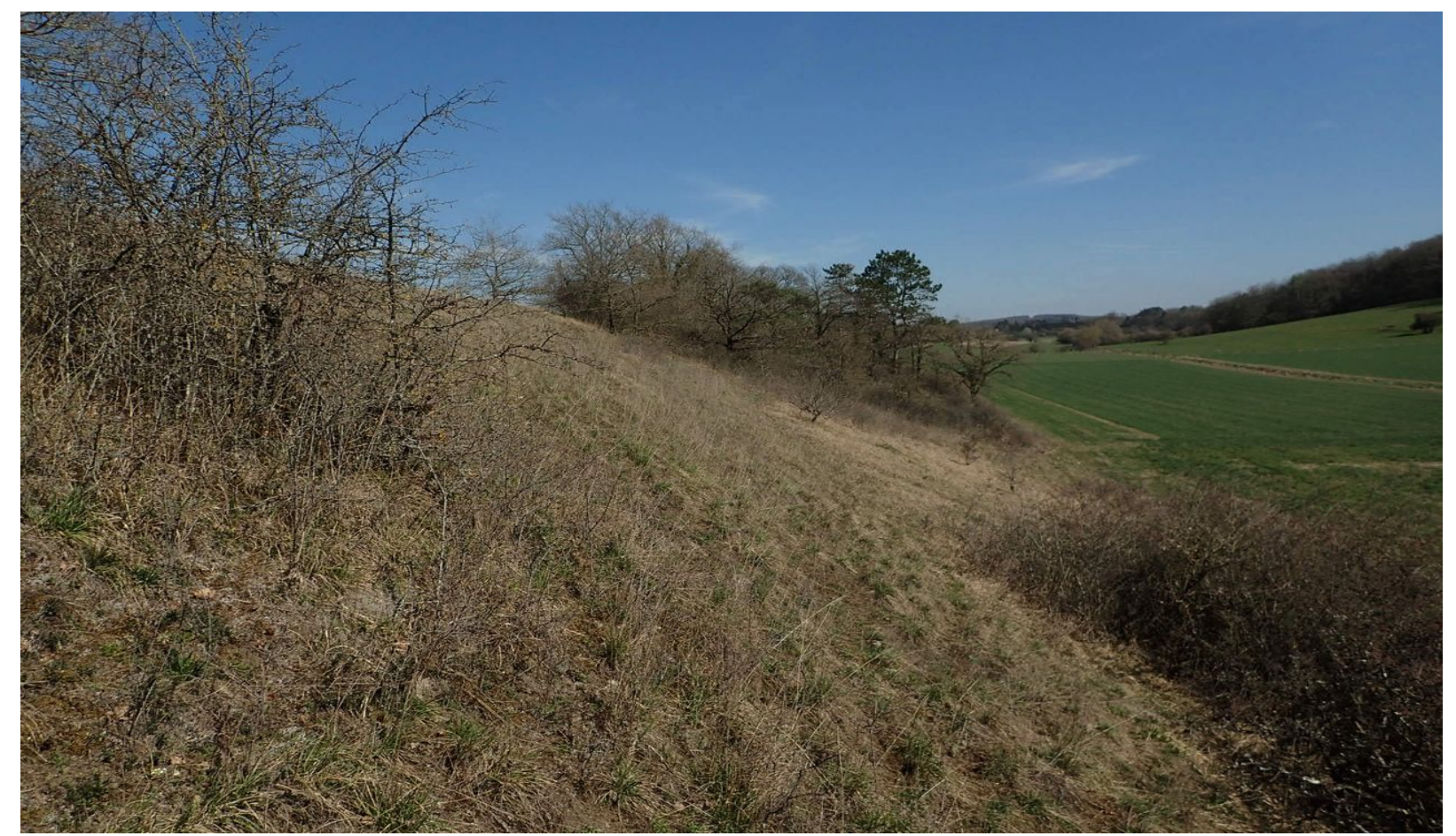

Figure 2: Aspect hivernal de l'habitat d'Hardya helgae à Eprave (pelouse ouest), 21.iii.2019 (photo J.-Y. Baugnée).

\footnotetext{
${ }^{2} \mathrm{http}: / /$ biodiversite. wallonie.be/fr/2383-tige-d-eprave.html?IDD=251659834\&IDC=1881
} 
Le troisième point d'observation (figure 3), localisé dans la partie centrale du site, au lieu-dit Les Gives, fait partie de la réserve naturelle domaniale dite du "Tige d'Eprave". Celle-ci est formée de deux parcelles agricoles contiguës s'étendant sur environ $150 \mathrm{~m}$ depuis la route jusqu'au rideau arboré à la base du versant en légère pente. La cicadelle a été récoltée dans la partie haute, la plus sèche, où Bromus erectus abonde aux côtés de Trifolium striatum L., Trisetum flavescens (L.) P. Beauv., Briza media L., Plantago media L., Ranunculus bulbosus L., Bromus hordeaceus L., Koeleria pyramidata (Lam.) P. Beauv., Poa pratensis subsp. angustifolia (L.) Dumort., Sanguisorba minor Scop., Sedum album L., Galium pumilum Murray, ...

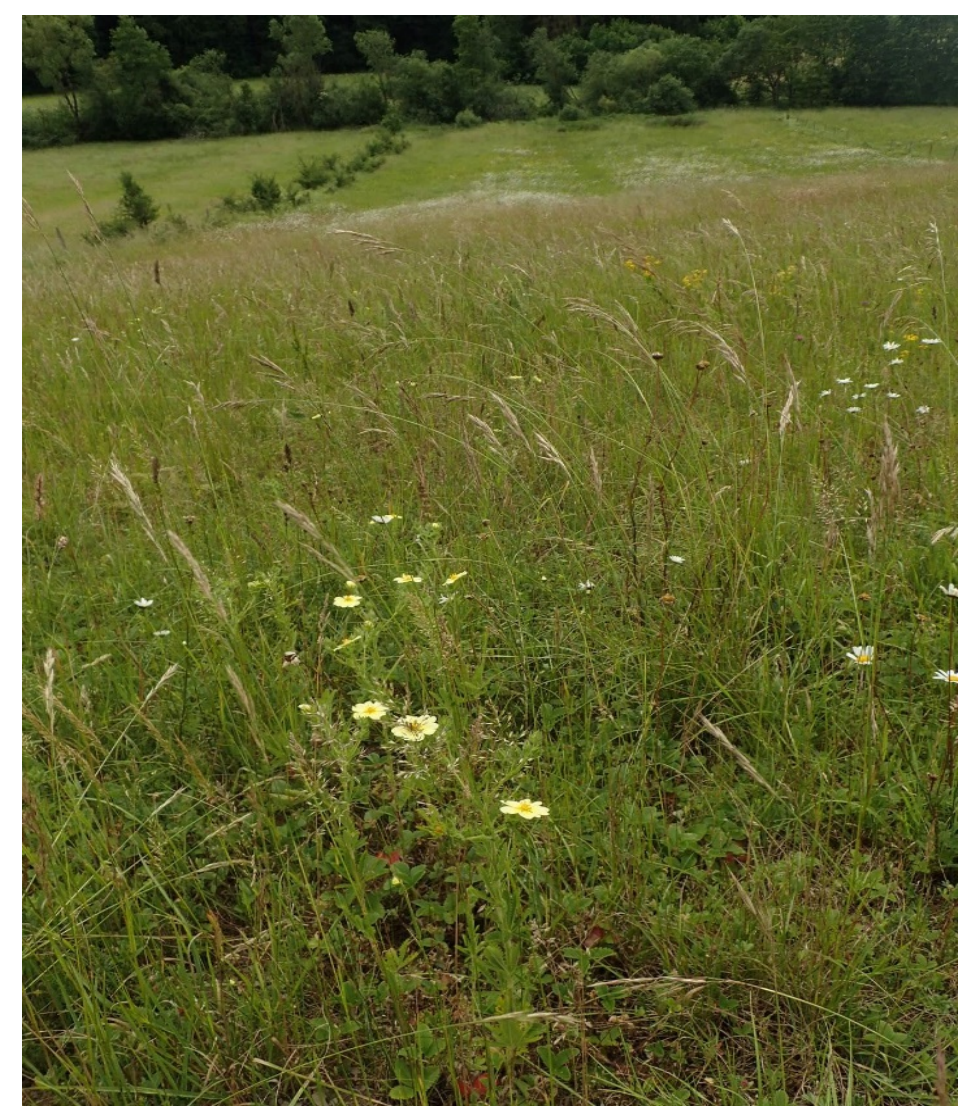

Figure 3: Aspect estival de l'habitat d'Hardya helgae à Eprave (RND), 14.vi.2019

(photo J.-Y. Baugnée).

D'après les cortèges floristiques relevés, ces trois stations correspondent largement à l'habitat E1.26 Pelouses calcaires mésophiles et méso-xérophiles dans la typologie WalEUNIS ${ }^{3}$.

Les autres Fulgoromorpha et Cicadomorpha recensés dans ces trois stations sont les Delphacidae Ditropsis flavipes (Signoret 1865), Ribautodelphax albostriatus (Fieber 1866) et Stenocranus minutus (Fabricius 1787), le Cercopidae Cercopis vulnerata Rossi 1807, l'Aphrophoridae Philaenus spumarius (Linnaeus 1758) et les Cicadellidae Psammotettix confinis (Dahlbom 1850), Turrutus socialis (Flor 1861), Zyginidia scutellaris (Herrich-Schäffer 1838), Artianus interstitialis (Germar 1821), Psammotettix helvolus (Kirschbaum 1868), Adarrus multinotatus (Boheman 1847), Thamnotettix dilutior (Kirschbaum 1868), Mocydia crocea (Herrich-Schäffer 1837), Zygina hyperici (Herrich-Schäffer 1836) et Goldeus harpago (Ribaut 1925).

\footnotetext{
${ }^{3}$ http://biodiversite.wallonie.be/fr/e1-26-pelouses-calcaires-mesophiles-et-meso-xerophiles.html?IDC=961
} 
Parmi ces espèces, relevons plus particulièrement Ditropsis flavipes, Delphacidae thermophile peu commun en Région wallonne et lié aussi à Bromus erectus, Goldeus harpago, cicadelle d'origine méridionale vivant sur Brachypodium pinnatum (s.l.) et atteignant sa limite nord européenne en FagneFamenne-Calestienne (Baugnée, 1995), ainsi que Artianus interstitialis, espèce liée aux pelouses et prairies sèches dont la présence en Belgique n'a semble-t-il jamais été documentée de manière formelle (plusieurs autres stations nous sont connues en Famenne).

\section{DISCUSSION}

Décrit tout récemment sur base de matériaux fournis issus de diverses localités du sud-ouest de l'Allemagne, Hardya helgae fut confondu auparavant avec H. tenuis (Nickel et al., 2017). Dans ce pays, les premières récoltes connues semblent remonter aux années 1980-1990 dans le Kaiserstuhl et l'Eifel. L'article original mentionne également du matériel du Grand-Duché de Luxembourg et de l'est de la France (Alsace). Ces occurrences rendaient donc fort plausible l'existence de $H$. helgae sur le territoire belge, d'autant que Bromus erectus, sa plante nourricière apparemment exclusive, est largement distribué sur les pelouses calcicoles wallonnes au sein des districts phytogéographiques mosan et lorrain (Lambinon \& Verloove, 2012). Dans ces conditions, H. helgae pourrait potentiellement être rencontré sur nombre d'autres sites moyennant des recherches spécifiques, d'autant que cette cicadelle se capture aisément au filet fauchoir et que sa phénologie (probablement bivoltine sous nos latitudes) est étendue, les adultes se rencontrant quasiment toute l'année.

En ce qui concerne l'Allemagne, Nickel et al. (2017) s'interrogent sur l'origine de leur espèce du fait de son absence dans les matériaux muséologiques antérieurs à 1980 et de son lien trophique étroit avec Bromus erectus, une plante fortement expansive depuis plusieurs décennies. Il a en effet été largement démontré que cette graminée pérenne, caractéristique des prairies et pelouses sèches calcicoles, montrait une tendance invasive dans différentes régions d'Europe centrale et occidentale, sans doute favorisée en cela par les changements des pratiques agro-pastorales et notamment l'abandon progressif du pâturage ovin au profit de la fauche, laquelle permet à ce brome de prendre le dessus sur ses concurrentes tels que Brachypodium pinnatum et d'autres (voir e.a. Schläpfer et al., 1998; Poniatowski et al., 2018). C'est donc en toute logique que Nickel et al. (2017) ont suggéré que la dissémination de $H$. helgae en Allemagne a suivi celle de sa plante hôte, une hypothèse d'ailleurs valable aussi pour Ditropsis flavipes.

Si la découverte de Hardya helgae à Rochefort constitue un évènement intéressant en soit sur le plan faunistique, elle doit être nuancée par le fait qu'une population fournie de cette cicadelle pourrait en fait refléter un certain état de dégradation des pelouses sèches! En effet, Poniatowski et al. (2018) ont démontré le lien entre l'expansion de Bromus erectus et l'appauvrissement du cortège floristique des pelouses sèches avec, conséquemment, une diminution de la diversité entomologique de cet habitat. Cela plaide pour une gestion différenciée faisant appel des méthodes variées au sein d'un même site, afin de favoriser l'hétérogénéité plutôt que l'uniformisation de la végétation (souvent engendrée par la fauche).

D'autre part, ces observations contribuent à mettre en lumière les nombreuses richesses biologiques du Tige d'Eprave, certes déjà connu pour son grand intérêt botanique (voir e.a. Limbourg, 2006) et lépidoptérologique, mais nettement moins parcouru par les naturalistes que d'autres endroits plus prestigieux de la région de Rochefort et Han-sur-Lesse. Les inventaires entomologiques en cours, orientés sur les Hémiptères et différentes familles de Diptères, Coléoptères et Hyménoptères, s'avèrent très prometteurs et ont déjà permis d'y noter diverses espèces rares ou d'intérêt particulier, incluant même quelques nouveautés pour la faune belge comme la cicadelle Artianus interstitialis déjà évoquée ci-avant, mais aussi l'abeille solitaire Osmia gallarum Spinola 1808 dont un mâle a été capturé en bordure de la réserve naturelle domaniale le 14.vi.2019 (leg. J.-Y. Baugnée et M. Paquay).

Quant à Hardya tenuis, son occurrence en Belgique repose sur une unique donnée déjà fort ancienne. C'est Fagel (1949) qui, le premier, cite cette espèce de Nettinne (province de Namur) d'après la collection de C. Van Volxem. Cette indication fut ensuite colportée dans les différents catalogues qui se sont succédés depuis lors (voir e.a. Van Stalle, 1989; Den Bieman et al., 2011) et, à notre connaissance, plus aucune observation de cette cicadelle n'a été documentée depuis. 
La collection Van Volxem, conservée à l'IRSNB, renferme effectivement deux spécimens femelles étiquetés "Nettinne", identifiés comme Hardya tenuis par H. Synave et collectés vraisemblablement au cours de la seconde moitié du $19^{\text {ème }}$ siècle (d'après la période d'activité de C. Van Volxem). A notre demande, J. Constant (IRSNB) a bien voulu réexaminer ces deux femelles sur base du travail de Nickel et al. (2017) et sa conclusion est qu'elles correspondent davantage à $H$. helgae, du fait de leur petite taille (3 $\mathrm{mm}$ ) et de leurs ailes antérieures ne dépassant pas l'extrémité de l'abdomen (J. Constant in litt., 18 janvier 2021).

Si une part d'incertitude demeure sur l'identité précise de ces deux spécimens (en l'absence de mâles associés), il paraît raisonnable d'exclure $H$. tenuis du catalogue belge en attendant un signalement attesté.

\section{REMERCIEMENTS}

Ce petit travail a bénéficié de l'aide précieuse de Jérôme Constant qui a accepté de réexaminer les Hardya de la collection de l'IRSNB. Les inventaires effectués au Tige d'Eprave ont été rendus agréables grâce à la participation de Marc Paquay et d'Emmanuelle Bisteau. Enfin, les échanges avec Herbert Nickel, Kees den Bieman et Dominik Poniatowski ont été également appréciés.

\section{BIBLIOGRAPHIE}

Baugnée J.-Y., 1995. Homoptères Auchénorhynques nouveaux pour la faune belge, récoltés dans le sud de l'EntreSambre-et-Meuse (Homoptera: Delphacidae, Cicadellidae). Bulletin et Annales de la Société royale belge d'Entomologie, 131, 161-167.

Biedermann, R. \& Niedringhaus R., 2004. Die Zikaden Deutschlands. Bestimmungstafeln für alle Arten. Wissenschaftlicher Akademischer Buchvertrieb Fründ, Scheeßel, iv +409 p.

Bourgoin T. \& Campbell B. C., 2002. Inferring a Phylogeny for Hemiptera: Falling into the 'Autapomorphic Trap'. Denisia, 4, 67-82.

Den Bieman K., Biedermann R., Nickel H. \& Niedringhaus R., 2011. The planthoppers and leafhoppers of Benelux. Cicadina - Supplement 1, WABV Fründ, 120 p.

Fagel G., 1949. Aperçu sur les connaissances actuelles des Jassides de Belgique (Hemiptera Homoptera). Bulletin et Annales de la Société Entomologique de Belgique, 85 (5/6), 144-153.

Forero D., 2008. The systematics of the Hemiptera. Revista Colombiana de Entomología, 34 (1), 1-21.

Garrouste R., 2015. Hémiptères de France, de Belgique, du Luxembourg et de Suisse. Guide Delachaux, Delachaux et Niestlé, $319 \mathrm{p}$.

Jach M., 2013. Fauna Europaea: Hemiptera Cicadomorpha. Fauna Europaea version 2017.06, http://www.faunaeu.org (20/11/2019)

Kunz G., Nickel H. \& Niedringhaus R., 2011. Fotoatlas der Zikaden Deutschlands. Photographic Atlas of the Planthoppers and Leafhoppers of Germany. WABV Fründ, 293 p.

Lambinon J. \& Verloove F., 2012. Nouvelle Flore de la Belgique, du Grand-Duché de Luxembourg, du Nord de la France et des Régions voisines (Ptéridophytes et Spermatophytes). Sixième édition. Meise, Jardin botanique national de Belgique, CXXXIX + 1195 p.

Limbourg P., 2006. Samedi 27 mai: prospection botanique à Eprave (I.F.B.: J6-24-21). Les Barbouillons, 230, 5355.

Lock K., 2019. Fifty leafhoppers new to Belgium (Hemiptera: Cicadellidae). Belgian Journal of Entomology, 88, 128.

Mühlethaler R., Holzinger W. E., Nickel H. \& Wachmann E., 2019. Die Zikaden Deutschlands, Osterreichs und der Schweiz. Quelle \& Meyer, 358 p.

Nickel H., 2003. The leafhoppers and planthoppers of Germany (Hemiptera, Auchenorrhyncha): patterns and strategies in a highly diverse group of phytophagous insects. Sofia and Moscow, $460 \mathrm{p}$. 
Nickel H., Holzinger W. E. \& Remane R., 2017. Hardya helgae nov. sp., a new leafhopper species from Central Europe (Hemiptera: Auchenorrhyncha: Cicadellidae). Linzer biologisher Beiträge, 49 (1), 707-719.

Poniatowski D., Hertenstein F., Raude N., Gottbehüt K., Nickel H. \& Fartmann T., 2018. The invasion of Bromus erectus alters species diversity of vascular plants and leafhoppers in calcareous grasslands. Insect Conservation and Diversity, 11, 578-586.

Remane R. \& Wachmann E., 1993. Zikaden. Kennenlernen - beobachten. Naturbuch Verlag, 288 p.

Schläpfer M., Zoller H. \& Körner C., 1998. Influences of mowing and grazing on plant species composition in calcareous grassland. Botanica Helvetica, 108, 57-67.

Van Stalle J., 1989. A Catalogue of Belgian Homoptera Auchenorrhyncha. Comptes rendus du Symposium "Invertébrés de Belgique", Institut royal des Sciences naturelles de Belgique, Bruxelles, p. 265-272.

Zahniser J. N. \& Dietrich C. H., 2013. A review of the tribes of Deltocephalinae (Hemiptera: Auchenorrhyncha: Cicadellidae). European Journal of Taxonomy, 45, 1-211.

(20 REF.) 